\title{
Plant breeding: a success story to be continued thanks to the advances in genomics
}

\author{
Jaime Prohens* \\ Instituto de Conservación y Mejora de la Agrodiversidad Valenciana, Universitat Politècnica de València, Valencia, Spain \\ *Correspondence: jprohens@btc.upv.es
}

Conventional plant breeding mostly relies upon the generation of new genetic combinations by sexual hybridization and the subsequent application of selection methods on phenotypically evaluated individuals and populations. This approach has been extremely efficient in providing a continuous supply of improved cultivars that have resulted in a dramatic increase of yield in most major crops. These yield increases have allowed time after time beating neo-Malthusian predictions that food production could not keep the pace of population growth in the twentieth century and beyond (Fedoroff, 2010). According to FAO data, in less than 50 years (1961-2009) the world average of cereal yields has increased from 1.35 to $3.51 \mathrm{t} / \mathrm{h}$. Thanks to these yield improvements, the total production of cereals has raised from $877 \cdot 10^{6}$ to $2489 \cdot 10^{6} \mathrm{t}$ with just an increase of $9 \%$ in this period in the acreage devoted to these staple crops. These unparalleled increases in yield have been possible thanks to improved cultivation techniques in combination with new cultivars. The new cultivars have been developed for adaptation to new management practices or growing conditions and vice versa, in a clear example to exploitation of genotype $\times$ environment $(\mathrm{G} \times \mathrm{E})$ interaction. One of the most recalled examples of the success of this approach comes from the so-called Green Revolution (Jain, 2010). The identification of dwarf and semi-dwarf genes in rice and wheat made possible the development of non-lodging cultivars with high yield in response to fertilization (Hedden, 2003). The adoption of the new cultivars combined with higher levels of fertilization resulted in dramatic yield increases in Southeast Asia (rice) and Mexico (wheat). Another illustration of the achievements of breeding involves maize in the US, where yields have increased by more than five-fold since 1930. These impressive yield increases have been preceded by the adoption of breeding innovations which, sequentially consisted on the selection within open-pollinated varieties, development of double and three-way hybrids, simple F1 hybrids, and GMO F1 hybrids (Hallauer and Carena, 2009). These success stories in the three major crops have a parallel in many other crops of different botanical families and with different uses.

The foundations of conventional breeding rely on: (a) the wide-scale use of diversity present in landraces and closely related species (primary and secondary gene pools), and (b) the development of breeding methods using Mendelian and quantitative genetics approaches for an efficient selection. With few exceptions the screening of available diversity and selection is based on phenotypic evaluation. The phenotype is the result of the expression of the genotype in a given environment, and this leads to the main limitations of conventional breeding. For traits with a polygenic inheritance and a high $\mathrm{G} \times \mathrm{E}$ interaction (e.g., yield), the efficiency of breeding programs will largely depend upon the detection and selection, based on phenotypic evaluation, of the appropriate genetic variants and adequate genetic combinations. Although phenotypic selection has frequently been successful to identify genes with a large effect on the phenotype, on many occasions favorable alleles of small effect, in particular for quantitative complex traits, have frequently remained unnoticed and have not been included in the gene pool used for breeding (Morgante and Salamini, 2003). Similarly, the masking effect of environment may reduce the efficiency of selection, resulting in the loss of favorable alleles during the selection process. As a consequence of these limitations of conventional breeding, additional genetic advances in major crops for yield or for other traits for which extensive breeding has been done is becoming increasingly difficult. In consequence, for some major crops the pace experimented for genetic gains in yield in the twentieth century will be difficult to be maintained if only existing conventional breeding technologies are used (Araus et al., 2008).

Fortunately, during the last decades many developments have taken place in molecular biology and genetic engineering. This has resulted in new tools and approaches for breeders that partially overcome the main limitations of genotype selection using exclusively phenotypic characterization. The development of a broad array of molecular markers has facilitated the identification of sources of variation and selection, in particular for complex traits or that were phenotypically difficult to evaluate. Also, the possibility of horizontal transfer of genes has resulted in genetically modified (GM) plants with new properties and characteristics. Despite the opposition of some sectors of the society and a tight legal scrutiny (Cunningham, 2003), GM crops have proved that can lead to a dramatic improvement of yield and quality without adverse effects on the environment or human health (Fedoroff, 2010). However, the greatest breeding advances that will be obtained in the near future will likely result from the applications of genomics to plant breeding. While genetics revolutionized plant breeding at the beginning of the twentieth century, genomics is leading to a new plant breeding revolution at the beginning of the twenty-first century. This is because genomics allows the direct study of the genotype and its relationship with the phenotype for a high number of genes and traits (Tester and Langridge, 2010), and therefore provides new tools and resources that are leading to a paradigm shift in the breeding approaches. Furthermore, genomic tools and approaches are widely accepted by the public opinion and in most cases free from criticism from social sectors reluctant to the use of genetic engineering in plant breeding.

Most of the new opportunities for breeders offered by the new genomics methodologies have resulted from the development of 
next generation sequencing (NGS) methods (Varshney et al., 2009), which make possible sequencing whole plant genomes or transcriptomes in a short period of time and at an increasingly reduced cost (Metzker, 2010). Genome or transcriptome sequences give breeders access to genes, their genomic position and function, as well as to large collections of markers that can be used for obtaining high density genetic maps, or for marker assisted selection (MAS; Tester and Langridge, 2010). The large amount of sequence reads poses a challenge to bioinformaticists, as it requires the development of software tools for the efficient processing in an automated way of the increasingly massive amount of genomic data produced.

An important contribution of advances in genomics for plant breeding comes from MAS, which relies in the linkage of the marker and gene or QTL of interest (Collard and Mackill, 2008). NGS sequencing projects produce large collections of markers, including intra-genic (or functional) markers which overcome the possibility of recombination between the functional gene or QTL and the marker (Metzker, 2010). The availability of markers spread throughout the genome facilitates introgression of individual or multiple genes or QTLs from one or several donors into the genetic background of a recurrent parent, as well as the gene pyramiding (i.e., the introduction of several genes or QTLs controlling traits of interest in a single genetic background). Another powerful application of MAS derived from the availability of a large number of markers is the "breeding by design" approach (Peleman and van der Voort, 2003), which predicts the outcome of a set of crosses on the basis of molecular markers information. A new application of MAS is genomic selection (GS), which is based on the simultaneous estimation of effects on the phenotype of all available loci, haplotypes, and markers without a previous selection of markers with effects on the phenotype (Jannink et al., 2010). GS requires availability of phenotypic and genotypic data and the establishment of a genetic model, so that the differences in the phenotype are explained by the markers analyzed (Lorenz et al., 2011).

Newly developed approaches arising from the development of genomics, like the combination of genetical genomics and bulked segregant analysis (BSA; Chen et al.,
2011), facilitates the identification of markers linked to genes. Availability of markers covering the whole genomic regions have made possible the development of special populations, like recombinant inbred lines (RILs), near isogenic lines (NILs), introgression lines (ILs), or chromosome segment substitution lines (CSSLs). These populations are contributing to increasing the accuracy in the detection of genes and QTLs and also represent a genetic material of great value for breeding programs. Also, association mapping, either using candidate gene association and genome-wide association (GWA; Zhu et al., 2008), which exploits the large number of recombination events occurred in the history of a germplasm collection, provide a better resolution of the genetic maps thanks to the availability of markers distributed throughout of the genome. Currently, approaches such as GWA, which avoids the limits of biparental populations, have also been adapted to the "breeding by design" approach.

Other genomic tools of interest that are being of great interest for breeders come from expression studies. Among them, the microarrays (Rensink and Buell, 2005) are particularly useful, as they allow studying the simultaneous expression of thousands of genes at a given time from a certain tissue from a plant subjected to specific conditions (stress, disease, etc.). Also, the detection of new alleles is facilitated using genomic approaches, either by direct sequencing and comparison with reference sequences (Varshney et al., 2009), or from the screening of either mutant or natural germplasm collections by means of targeting induced local lesions in genomes (TILLING) or ecotype TILLING (EcoTILLING) methodologies (Till et al., 2003; Comai et al., 2004).

At present, many genomics resources and tools are already available to breeders, and present and future advances in the field of genomics will be the key to another giant leap in plant breeding. For example, the so-called super domestication, which has been defined as "the processes that lead to a domesticate with dramatically increased yield that could not be selected in natural environments from naturally occurring variation without recourse to new technologies" (Vaughan et al., 2007), requires from genomics tools and approaches. Genomics is also helping conventional breeding in achieving important advances in the breeding of crops that from the point of view of genetic improvement have remained as orphan or neglected (Varshney et al., 2010). Therefore, while conventional plant breeding has been, is, and will be successful at improving our crops, the application of genomic approaches, tools, and resources to practical plant breeding will push forward the genetic gains obtained by breeding programs in a precision breeding approach (Collard and Mackill, 2008).

Plant breeding is a highly dynamic science that has experienced a tremendous development during the last century. The development of genomics is allowing the incorporation of new tools and resources to address the important new challenges for agriculture. These include restriction in the availability of new lands, deterioration of some of the agricultural environments, and the need of more environment friendly and sustainable agricultural practices in a climate change context. The development of genomics is and will be of great help to plant breeders for exploiting the natural or artificially created diversity for developing new cultivars with improved characteristics that will allow coping successfully with the present and future challenges in a new Green Revolution.

\section{ACKNOWLEDGMENTS}

This work has been funded by the Ministerio de Ciencia y Tecnología (AGL2009-07257), Generalitat Valenciana (ACOMP/2011/032), and Universitat Politècnica de València (PAID-05-10-2318).

\section{REFERENCES}

Araus, J.L., Slafer, G. A., Royo, C., and Serret, M.D. (2008). Breeding for yield potential and stress adaptation in cereals. Crit. Rev. Plant Sci. 27, 377-412.

Chen, X., Hedley, P. E., Morris, J., Liu, H., Niks, R. E., and Waugh, R. (2011). Combining genetical genomics and bulked segregant analysis-based differential expression: an approach to gene localization. Theor. Appl. Genet. 122, 1375-1383.

Collard, B. C.Y., and Mackill, D. J. (2008). Marker-assisted selection: an approach for precision plant breeding in the twenty-first century. Philos. Trans. R. Soc. B 363, 557-572.

Comai, L., Young, K., Till, B. J., Reynolds, S. H., Greene, E. A., Codomo, C. A., Enns, L. C., Johnson, J. E., Burtner C., Odden, A. R., and Henikoff, S. (2004). Efficient discovery of DNA polymorphisms in natural populations by EcoTILLING. Plant J. 37, 778-786.

Cunningham, P. (2003). "Public understanding and perception of plant biotechnology in the EU: myth and reality," in In the Wake of the Double Helix: from the 
Green Revolution to the Gene Revolution, eds R. R. Tuberosa, R. L. Phillips, and M. Gale (Bologna, Italy: Avenue Media), 617-625.

Fedoroff,N.V.(2010). The past, present and future of crop genetic modification. New Biotechnol. 27, 461-465.

Hallauer, A. R., and Carena, M. J. (2009). "Maize breeding," in Handbook of Plant Breeding: Cereals, ed. M. J. Carena (New York, NY: Springer), 3-98.

Hedden, P. (2003). The genes of Green Revolution. Trends Plant Sci. 19, 5-9.

Jain, H. K. (2010). The Green Revolution: History, Impact and Future. Houston, TX: Studium Press.

Jannink, J. L., Lorenz, A. J., and Iwata, H. (2010). Genomic selection in plant breeding: from theory to practice. Brief. Funct. Genomics 9, 166-177.

Lorenz, A. J., Chao, S., Asoro, F. G., Heffner, E. L., Hayashi, T., Iwata, H., Smith, K. P., Sorrells, M. K., and Jannink, J. L. (2011). Genomic selection in plant breeding: knowledge and prospects. Adv. Agron. 110, 77-123.

Metzker, M. (2010). Sequencing technologies-the next generation. Nat. Rev. Genet. 11, 31-46.
Morgante, M., and Salamini, F. (2003). From plant genomics to breeding practice. Curr. Opin. Biotechnol. 14, 214-219.

Peleman, J. D., and van der Voort, J. R. (2003). Breeding by design. Trends Plant Sci. 8, 330-334.

Rensink, W. A., and Buell, C. R. (2005). Microarray expression profiling resources for plant genomics. Trends Plant Sci. 10, 603-609.

Tester, M., and Langridge, P. (2010). Breeding technologies to increase crop production in a changing world. Science 327, 818-822.

Till, B. J., Reynolds, S. H., Greene, E. A., Codomo, C. A., Enns, L. C., Johnson, J. E., Burtner, C., Odden, A. R., Young, K., Taylor, N. E., Henikoff, J. G., Comai, L., and Henikoff, S. (2003). Large-scale discovery of induced point mutations with high-throughput TILLING. Genome Res. 13, 524-530.

Varshney, R. K., Glaszmann, J. C., Leung, H., and Ribaut, J.M. (2010). More genomic resources for less-studied crops. Trends Biotechnol. 28, 452-460.

Varshney, R. K., Nayak, S. N., May, G. D., and Jackson, S. A. (2009). Next-generation sequencing technologies and their implications for crop genetics and breeding Trends Biotechnol. 27, 522-530.

Vaughan, D. A., Balász, E., and Heslop-Harrison, J. S. (2007). From crop domestication to super-domestication. Ann. Bot. 100, 893-901.

Zhu, C., Gore, M., Buckler, E. S., and Yu, J. (2008). Status and prospects of association mapping in plants. Plant Genome 1, 5-20.

Received: 19 August 2011; accepted: 29 August 2011; published online: 16 September 2011.

Citation: Prohens J (2011) Plant breeding: a success story to be continued thanks to the advances in genomics. Front. Plant Sci. 2:51. doi: 10.3389/fpls.2011.00051

This article was submitted to Frontiers in Crop Science and Horticulture, a specialty of Frontiers in Plant Science. Copyright (c) 2011 Prohens. This is an open-access article subject to a non-exclusive license between the authors and Frontiers Media SA, which permits use, distribution and reproduction in other forums, provided the original authors and source are credited and other Frontiers conditions are complied with. 